\title{
Characterization of diffeomorphisms that are symplectomorphisms
}

\author{
by \\ Stanisław Janeczko and Zbigniew Jelonek (Warszawa)
}

\begin{abstract}
Let $\left(X, \omega_{X}\right)$ and $\left(Y, \omega_{Y}\right)$ be compact symplectic manifolds (resp. symplectic manifolds) of dimension $2 n>2$. Fix $0<s<n$ (resp. $0<k \leq n$ ) and assume that a diffeomorphism $\Phi: X \rightarrow Y$ maps all $2 s$-dimensional symplectic submanifolds of $X$ to symplectic submanifolds of $Y$ (resp. all isotropic $k$-dimensional tori of $X$ to isotropic tori of $Y$ ). We prove that in both cases $\Phi$ is a conformal symplectomorphism, i.e., there is a constant $c \neq 0$ such that $\Phi^{*} \omega_{Y}=c \omega_{X}$.
\end{abstract}

1. Introduction and main results. Let $\left(X, \omega_{0}\right)$ be the standard symplectic vector space over $\mathbb{R}$ of dimension $2 n$, i.e., $X \cong \mathbb{R}^{2 n}$ and $\omega_{0}=$ $\sum_{i} d x_{i} \wedge d y_{i}$ is the standard non-degenerate skew-symmetric form on $X$. The group of automorphisms of $\left(X, \omega_{0}\right)$ is called the symplectic group and is denoted by $\mathbf{S p}(X)$.

Linear symplectomorphisms of $\left(X, \omega_{0}\right)$ are characterized in [6] as linear automorphisms of $X$ preserving some minimal, complete data defined by $\omega_{0}$ on systems of linear subspaces. In this way the linear symplectic group $\mathbf{S p}(X)$ may be characterized geometrically together with its natural conformal and anti-symplectic extensions. It is the natural task to put the linear considerations into a more general nonlinear context (cf. [5], [7]).

Let $\left(X, \omega_{X}\right)$ and $\left(Y, \omega_{Y}\right)$ be symplectic manifolds of dimension $2 n$ (all manifolds in this paper are assumed to be smooth and connected). We say that a diffeomorphism $F: X \rightarrow Y$ is a conformal symplectomorphism (cf. [10]) if there is a non-zero constant $c \in \mathbb{R}$ such that $F^{*} \omega_{Y}=c \omega_{X}$.

Here is our main result:

THEOREM 1. Let $\left(X, \omega_{X}\right)$ and $\left(Y, \omega_{Y}\right)$ be symplectic manifolds of dimension $2 n>2$ (resp. compact symplectic manifolds of dimension $2 n>2$ ). Fix a number $1<k \leq n$ (resp. $0<s<n)$. Assume that $\Phi: X \rightarrow Y$ is a diffeomorphism which transforms all $k$-dimensional isotropic tori of $X$ (resp. 
$2 s$-dimensional symplectic, closed submanifolds of $X$ ) onto isotropic tori of $Y$ (resp. symplectic, closed submanifolds of $Y$ ). Then $\Phi$ is a conformal symplectomorphism.

In other words, for any fixed $k$ (or $s$ ) as above, the conformal symplectic structure on $X$ is uniquely determined by the family of all $k$-dimensional isotropic tori (resp. $2 s$-dimensional, closed symplectic submanifolds) of $X$. The proof is based on a number of results which we state below.

First we show that any element of $\mathbf{S p}\left(\mathbb{R}^{2 n}, \omega_{0}\right)$ can be finitely decomposed into elements of a family of elementary automorphisms.

TheOREM 2. The symplectic group $\mathbf{S p}\left(\mathbb{R}^{2 n}, \omega_{0}\right)$ is generated by the family of elementary symplectic automorphisms:

$$
\left\{L_{i}\left(c_{i}\right), L_{i j}\left(c_{i j}\right), R_{i}\left(d_{i}\right), R_{i j}\left(d_{i j}\right): 0<i<j \leq n, c_{i}, c_{i j}, d_{i}, d_{i j} \in \mathbb{R}\right\}
$$

defined by

$L_{i}\left(c_{i}\right)\left(x_{1}, \ldots, x_{n}, y_{1}, \ldots, y_{n}\right)=\left(x_{1}, \ldots, x_{n}, y_{1}, \ldots, y_{i-1}, y_{i}+c_{i} x_{i}, y_{i+1}, \ldots, y_{n}\right)$,

$L_{i j}\left(c_{i j}\right)\left(x_{1}, \ldots, x_{n}, y_{1}, \ldots, y_{n}\right)$

$=\left(x_{1}, \ldots, x_{n}, y_{1}, \ldots, y_{i-1}, y_{i}+c_{i j} x_{j}, y_{i+1}, \ldots, y_{j-1}, y_{j}+c_{i j} x_{i}, y_{j+1}, \ldots, y_{n}\right)$, $R_{i}\left(d_{i}\right)\left(x_{1}, \ldots, x_{n}, y_{1}, \ldots, y_{n}\right)=\left(x_{1}, \ldots, x_{i-1}, x_{i}+d_{i} y_{i}, x_{i+1}, \ldots, x_{n}, y_{1}, \ldots, y_{n}\right)$, $R_{i j}\left(d_{i j}\right)\left(x_{1}, \ldots, x_{n}, y_{1}, \ldots, y_{n}\right)$ $=\left(x_{1}, \ldots, x_{i-1}, x_{i}+d_{i j} y_{j}, x_{i+1}, \ldots, x_{j-1}, x_{j}+d_{i j} y_{i}, x_{j+1}, \ldots, x_{n}, y_{1}, \ldots, y_{n}\right)$.

Let $H: \mathbb{R}^{2 n} \times \mathbb{R} \ni(z, t) \mapsto H(z, t) \in \mathbb{R}$ be a smooth function and consider the system of differential equations (Hamiltonian system)

$$
\frac{d}{d t} \phi(t, z)=J_{0}\left(\nabla_{z} H\right)(\phi(t, x), t), \quad \phi(0, z)=z,
$$

where $z=\left(x_{1}, \ldots, x_{n}, y_{1}, \ldots, y_{n}\right)$ and $J_{0}$ is the $2 n \times 2 n$ matrix of $\omega_{0}$. Then for the smooth solution $\phi(t, z)$ the map $\Phi(z)=\phi(1, z)$ is a diffeomorphism preserving $\omega_{0}$, i.e. a symplectomorphism, which is called the hamiltonian symplectomorphism with Hamiltonian $H$ (cf. [11]). The next basic result we get is

TheOREM 3. For any linear symplectomorphism $L:\left(\mathbb{R}^{2 n}, \omega_{0}\right) \rightarrow\left(\mathbb{R}^{2 n}, \omega_{0}\right)$ there exists a polynomial Hamiltonian

$$
H_{L}(z, t)=\sum_{i, j=1}^{2 n} a_{i, j}(t) z_{i} z_{j},
$$

where $a_{i, j}(t) \in \mathbb{R}[t]$ are polynomials of $t$. Moreover, $H_{L}$ can be computed effectively.

Recall that a submanifold $Z$ of a symplectic manifold $X$ is isotropic if $\left.\omega\right|_{T Z}=0$. We will call $Z$ a symplectic submanifold of $X$ if it is closed and the 
pair $\left(Z,\left.\omega\right|_{T Z}\right)$ is a symplectic manifold. Existence of isotropic or symplectic submanifolds with prescribed data in a compact symplectic manifold is a fundamental geometric property of symplectic structures (cf. [3]). In fact, we can find such submanifolds which satisfy some extra conditions:

THEOREM 4. Let $(X, \omega)$ be a symplectic manifold of dimension $2 n$ (resp. compact symplectic manifold of dimension $2 n$ ). Let $a_{1}, \ldots, a_{m}$ be a family of points of $X$. Take $0<k \leq n($ resp. $0<s \leq n)$. For every $i=1, \ldots, m$ choose a linear $k$-dimensional isotropic subspace (resp. a $2 s$-dimensional symplectic subspace) $H_{i} \subset T_{a_{i}} X$. Then there is a closed isotropic $k$-dimensional torus (resp. a closed symplectic 2 s-dimensional submanifold) $Y \subset X$ such that

$$
a_{i} \in Y, \quad T_{a_{i}} Y=H_{i}, \quad i=1, \ldots, m .
$$

The purpose of this article is twofold: First, we provide the new results in still basic linear symplectic geometry, formulated above. Second, we characterize general symplectic manifolds and their structure groups through the family of isotropic or symplectic submanifolds and their basic invariance. This leads to a complete geometric characterization of symplectomorphisms and to a reinterpretation of symplectomorphisms as diffeomorphisms acting purely on isotropic or symplectic submanifolds (cf. [2], [9]). We prove an isotropic version of our results for isotropic tori as a smallest optimal (complete for the invariance considered) family of isotropic submanifolds. The corresponding invariance result on symplectic submanifolds of a compact symplectic manifold, following from the main theorem, reads as follows.

THEOREM 5. Let $(X, \omega)$ be a compact symplectic manifold of dimension $2 n>2$. Fix a number $0<k<n$. Assume that $\Phi: X \rightarrow X$ is a diffeomorphism which maps all $2 k$-dimensional symplectic submanifolds of $X$ onto submanifolds of the same type. Then $\Phi$ is a symplectomorphism or an antisymplectomorphism, i.e., $\Phi^{*} \omega= \pm \omega$. If $\Phi$ preserves orientation and $n$ is odd, then $\Phi$ is a symplectomorphism. Moreover, if $n$ is even, then $\Phi$ has to preserve orientation.

2. Generators of the group $\operatorname{Sp}\left(\mathbb{R}^{2 n}\right)$. Here we recall some basic facts about the linear symplectic group. Let $(X, \omega)$ be a symplectic vector space. There exists a basis of $X$, called a symplectic basis, $u_{1}, \ldots, u_{n}, v_{1}, \ldots, v_{n}$, such that

$$
\omega\left(u_{i}, u_{j}\right)=\omega\left(v_{i}, v_{j}\right)=0, \quad \omega\left(u_{i}, v_{j}\right)=\delta_{i j} .
$$

Let $\left(X, \omega_{X}\right)$ and $\left(Y, \omega_{Y}\right)$ be symplectic vector spaces. We say that a linear isomorphism $F: X \rightarrow Y$ is a symplectomorphism (or is symplectic on $X)$ if $F^{*} \omega_{Y}=\omega_{X}$, i.e., $\omega_{X}(x, y)=\omega_{Y}(F(x), F(y))$ for every $x, y \in X$. The group of symplectomorphisms of $(X, \omega)$ is called the symplectic group and is denoted by $\mathbf{S p}(X, \omega)$. Via a symplectic basis, $X$ can be identified with 
the standard symplectic space $\left(\mathbb{R}^{2 n}, \omega_{0}\right)$ and $\mathbf{S p}(X, \omega)$ can be identified with the group of $2 n \times 2 n$ real matrices $A$ which satisfy $A^{T} J_{0} A=J_{0}$, where $J_{0}$ is the $2 n \times 2 n$ matrix of $\omega_{0}$ (in the standard basis), i.e.,

$$
J_{0}=\left[\begin{array}{cccccc}
0 & \ldots & 0 & -1 & \ldots & 0 \\
\vdots & & \vdots & \vdots & & \vdots \\
0 & \ldots & 0 & 0 & \ldots & -1 \\
1 & \ldots & 0 & 0 & \ldots & 0 \\
\vdots & & \vdots & \vdots & & \vdots \\
0 & \ldots & 1 & 0 & \ldots & 0
\end{array}\right]
$$

We can define the following "elementary" symplectomorphisms: $L_{i}\left(c_{i}\right)\left(x_{1}, \ldots, x_{n}, y_{1}, \ldots, y_{n}\right)=\left(x_{1}, \ldots, x_{n}, y_{1}, \ldots, y_{i-1}, y_{i}+c_{i} x_{i}, y_{i+1}, \ldots, y_{n}\right)$, $L_{i j}\left(c_{i j}\right)\left(x_{1}, \ldots, x_{n}, y_{1}, \ldots, y_{n}\right)$ $=\left(x_{1}, \ldots, x_{n}, y_{1}, \ldots, y_{i-1}, y_{i}+c_{i j} x_{j}, y_{i+1}, \ldots, y_{j-1}, y_{j}+c_{i j} x_{i}, y_{j+1}, \ldots, y_{n}\right)$, $R_{i}\left(d_{i}\right)\left(x_{1}, \ldots, x_{n}, y_{1}, \ldots, y_{n}\right)=\left(x_{1}, \ldots, x_{i-1}, x_{i}+d_{i} y_{i}, x_{i+1}, \ldots, x_{n}, y_{1}, \ldots, y_{n}\right)$, $R_{i j}\left(d_{i j}\right)\left(x_{1}, \ldots, x_{n}, y_{1}, \ldots, y_{n}\right)$ $=\left(x_{1}, \ldots, x_{i-1}, x_{i}+d_{i j} y_{j}, x_{i+1}, \ldots, x_{j-1}, x_{j}+d_{i j} y_{i}, x_{j+1}, \ldots, x_{n}, y_{1}, \ldots, y_{n}\right)$, where $c_{i}, c_{i j}, d_{i}, d_{i j}$ are real numbers and $1 \leq i<j \leq n$.

We have the following basic result:

THeOREM 2.1. Let $X=\left(\mathbb{R}^{2 n}, \omega_{0}\right)$ be the standard symplectic vector space. Then the group $\mathbf{S p}(X)$ is generated by the following family of elementary symplectomorphisms:

$$
\left\{L_{i}\left(c_{i}\right), L_{i j}\left(c_{i j}\right), R_{i}\left(d_{i}\right), R_{i j}\left(d_{i j}\right): 0<i<j \leq n \text { and } c_{i}, c_{i j}, d_{i}, d_{i j} \in \mathbb{R}\right\},
$$

i.e. if $g \in \mathbf{S p}(X)$ then $g=\prod_{i=1}^{m} e_{i}$, where $e_{i}$ is one of the elementary symplectomorphisms and $m \in \mathbb{N}$.

Proof. We reason by induction. For $n=1$ we have $\mathbf{S p}\left(\mathbb{R}^{2}\right)=\mathbf{S L}(2)$ and the result is well known from linear algebra. Assume $n>1$.

Let $S: \mathbb{R}^{2 n} \rightarrow \mathbb{R}^{2 n}$ be a linear symplectomorphism. Denote the coordinates by $x_{1}, \ldots, x_{n}, y_{1}, \ldots, y_{n}\left(\right.$ where $\left.\omega_{0}=\sum_{i} d x_{i} \wedge d y_{i}\right)$. We have

$$
S\left(x_{1}, y_{1}, \ldots, x_{n}, y_{n}\right)=\left(\sum_{i} a_{1 i} x_{i}+\sum_{j} b_{1 j} y_{j}, \ldots, \sum_{i} a_{2 n, i} x_{i}+\sum_{j} b_{2 n, j} y_{j}\right) .
$$

Observe how the rows of the matrix of $S$ are transformed under the composition $S \circ L$ with an elementary symplectomorphism $L$ (for simplicity we consider only the first row). After composition with $L_{i}(c)$ we have

$$
\left(a_{11}, \ldots, a_{1 n}, b_{11}, \ldots, b_{1 n}\right) \mapsto\left(a_{11}, \ldots, a_{1 i}+c b_{1 i}, \ldots, a_{1 n}, b_{11}, \ldots, b_{1 n}\right),
$$


with $L_{i j}(c)$ we have

$$
\begin{aligned}
\left(a_{11}, \ldots,\right. & \left.a_{1 n}, b_{11}, \ldots, b_{1 n}\right) \\
& \mapsto\left(a_{11}, \ldots, a_{1 i}+c b_{1 j}, \ldots, a_{1 j}+c b_{1 i}, \ldots, a_{1 n}, b_{11}, \ldots, b_{1 n}\right),
\end{aligned}
$$

with $R_{i}(c)$ we have

$$
\left(a_{11}, \ldots, a_{1 n}, b_{11}, \ldots, b_{1 n}\right) \mapsto\left(a_{11}, \ldots, a_{1 n}, b_{11}, \ldots, b_{1 i}+c a_{1 i}, \ldots, b_{1 n}\right),
$$

and with $R_{i j}(c)$ we have

$$
\begin{aligned}
\left(a_{11}, \ldots,\right. & \left.a_{1 n}, b_{11}, \ldots, b_{1 n}\right) \\
& \mapsto\left(a_{11}, \ldots, a_{1 n}, b_{11}, \ldots, b_{1 i}+c a_{1 j}, \ldots, b_{1 j}+c a_{1 i}, \ldots, b_{1 n}\right) .
\end{aligned}
$$

Transformations (1)-(4) will be called elementary operations. Now we show that using only elementary operations we can transform the first row of $S$ into $(1,0, \ldots, 0)$ and the second into $(0, \ldots, 0,1,0, \ldots, 0)$ (here the unit corresponds to $\left.b_{1 n}\right)$.

First note that the rows $\mathbf{r}_{1}, \ldots, \mathbf{r}_{2 n}$ of the matrix $S$ form a symplectic basis. Now, consider the first row. Of course it has a non-zero element, say $b_{1 s}$. Using $L_{s}(c)$ we can assume that also $a_{1 s} \neq 0$. Now using $L_{i s}(c)$ and $R_{j s}(d)$ for sufficiently general $c$ and $d$ we can assume that all elements of the first row are non-zero. Again applying $R_{i}(c)$ for $i>1$ we can now transform the first row into $\left(a_{11}, \ldots, a_{1 n}, 1,0, \ldots, 0\right)$. Using $L_{1 j}(c)$ we can transform this row into $(1,0, \ldots, 0,1,0, \ldots, 0)$ and finally using $R_{1}(-1)$ we obtain $(1,0, \ldots, 0)$. Now consider the row $\mathbf{r}_{n+1}$ (after these transformations): $\mathbf{r}_{n+1}=\left(a_{n+1,1}, \ldots, a_{n+1, n}, b_{n+1,1}, \ldots, b_{n+1, n}\right)$. We can apply our method to the subrow $\left(a_{n+1,2}, \ldots, a_{n+1, n}, b_{n+1,2}, \ldots, b_{n+1, n}\right)$ (if it is non-zero) and finally obtain the row $\left(a_{n+1,1}, 1,0, \ldots, 0, b_{n+1,1}, 0, \ldots, 0\right)$ (or $\left(a_{n+1,1}, 0, \ldots\right.$ $\left.\left.\ldots, 0, b_{n+1,1}, 0, \ldots, 0\right)\right)$. Since the value of $\omega_{0}$ on these two rows is 1 we conclude that $b_{n+1,1}=1$. Now (in the first case) we can use $L_{12}(-1)$ to obtain a row of the form $\left(a_{n+1,1}, 0, \ldots, 0,1,0, \ldots, 0\right)$. Finally, applying $L_{1}\left(-a_{12}\right)$ we get $(0, \ldots, 0,1,0, \ldots, 0)$.

Thus under all these compositions the matrix of $S$ has the form

$$
\left[\begin{array}{ccccccc}
1 & 0 & 0 & \ldots & 0 & \ldots & 0 \\
a_{21} & a_{22} & a_{23} & \ldots & b_{21} & \ldots & b_{2 n} \\
a_{31} & a_{32} & a_{33} & \ldots & b_{31} & \ldots & b_{3 n} \\
\vdots & \vdots & \vdots & \vdots & \vdots & \vdots & \vdots \\
0 & 0 & 0 & \ldots & 1 & \ldots & 0 \\
a_{n+1,1} & a_{n+1,2} & a_{n+1,3} & \ldots & b_{n+1,1} & \ldots & b_{n+1, n} \\
a_{n+2,1} & a_{n+2,2} & a_{n+2,3} & \ldots & b_{n+2,1} & \ldots & b_{4 n} \\
\vdots & \vdots & \vdots & \vdots & \vdots & \vdots & \vdots \\
a_{2 n, 1} & a_{2 n, 2} & a_{2 n, 3} & \ldots & b_{2 n, 1} & \ldots & b_{2 n, n}
\end{array}\right]
$$


For $j \neq 1, n+1$ we have $\omega_{0}\left(\mathbf{r}_{1}, \mathbf{r}_{j}\right)=0$ and $\omega_{0}\left(\mathbf{r}_{n+1}, \mathbf{r}_{j}\right)=0$. We can easily conclude that for all such $j$ the elements $a_{j 1}$ and $b_{j 1}$ in $S$ are 0 . This implies that the matrix

$$
\left[\begin{array}{cccccc}
a_{22} & a_{23} & \ldots & b_{22} & \ldots & b_{2 n} \\
a_{32} & a_{33} & \ldots & b_{32} & \ldots & b_{3 n} \\
\vdots & \vdots & \vdots & \vdots & \vdots & \vdots \\
a_{n 2} & a_{n 3} & \ldots & b_{n 2} & \ldots & b_{n n} \\
a_{n+2,2} & a_{n+2,3} & \ldots & b_{n+2,2} & \ldots & b_{n+2, n} \\
\vdots & \vdots & \vdots & \vdots & \vdots & \vdots \\
a_{2 n, 2} & a_{2 n, 3} & \ldots & b_{2 n, 2} & \ldots & b_{2 n, n}
\end{array}\right]
$$

is a symplectic matrix and we can apply the induction hypothesis.

We conclude this section by recalling (and extending) some result from $[6]$.

Definition 2.2. Let $\mathcal{A}_{l, 2 r} \subset G(l, 2 n)$ denote the set of all $l$-dimensional linear subspaces of $X$ on which the form $\omega$ has rank $\leq 2 r$.

Of course $\mathcal{A}_{l, 2 r} \subset \mathcal{A}_{l, 2 r+2}$ if $2 r+2 \leq l$. We have the following important (see [6, Theorem 6.2]):

THEOREM 2.3. Let $(X, \omega)$ be a symplectic vector space of dimension $2 n$ and let $F: X \rightarrow X$ be a linear automorphism. Let $0<2 r<2 n$. Assume $F$ maps $\mathcal{A}_{2 r, 2 r-2}$ into $\mathcal{A}_{2 r, 2 r-2}$. Then there is a non-zero constant c such that $F^{*} \omega=c \omega$.

From Theorem 2.3 we can deduce the following interesting facts:

Proposition 2.4. Let $\left(X, \omega_{X}\right)$ and $\left(Y, \omega_{Y}\right)$ be symplectic vector spaces of dimension $2 n$ and let $F: X \rightarrow Y$ be a linear isomorphism. Fix a number $s$ with $0<s<n$ and assume that $F$ maps all $2 s$-dimensional symplectic subspaces of $X$ onto symplectic subspaces of $Y$. Then there is a non-zero constant $c$ such that $F^{*} \omega_{Y}=c \omega_{X}$.

Proof. Via a symplectic basis we can assume that $\left(X, \omega_{X}\right) \cong\left(\mathbb{R}^{2 n}, \omega_{0}\right) \cong$ $\left(Y, \omega_{Y}\right)$. By assumption the mapping $F^{*}$ induced by $F$ maps the set $A=$ $\mathcal{A}_{2 s, 2 s} \backslash \mathcal{A}_{2 s, 2 s-2}$ into $A$. Of course $F^{*}: A \rightarrow A$ is an injection. Since $A$ is a smooth algebraic variety and $F^{*}$ is regular, the Borel Theorem (see [1]) implies that $F^{*}$ is a bijection. This means that $F$ maps $\mathcal{A}_{2 s, 2 s-2}$ into the same set, and we conclude the proof by applying Theorem 2.3.

Proposition 2.5. Let $\left(X, \omega_{X}\right)$ and $\left(Y, \omega_{Y}\right)$ be symplectic vector spaces of dimension $2 n$ and let $F: X \rightarrow Y$ be a linear isomorphism. Fix a number $k$ with $1<k \leq n$ and assume that $F$ maps all $k$-dimensional isotropic sub- 
spaces of $X$ onto isotropic subspaces of $Y$. Then there is a non-zero constant c such that $F^{*} \omega_{Y}=c \omega_{X}$.

Proof. For $k=2$ this follows immediately from Theorem 2.3. Assume that $k>2$. Take a plane $H$ belonging to $\mathcal{A}_{2,0}$. Since $H$ is isotropic, we can extend $H$ to a $k$-dimensional isotropic subspace $L$. By the assumption, $L$ is mapped onto an isotropic subspace $F(L)$. Observe that $F(H)$ is contained in $F(L)$, so $F(H)$ is also isotropic. In particular, $F(H) \in \mathcal{A}_{2,0}$. Then Theorem 2.3 yields the statement.

We end this section with:

Proposition 2.6. Let $X$ be a vector space of dimension $2 n$ and let $\omega_{1}, \omega_{2}$ be two symplectic forms on $X$. If $\mathbf{S p}\left(X, \omega_{1}\right) \subset \mathbf{S p}\left(X, \omega_{2}\right)$, then there exists a non-zero constant $c$ such that $\omega_{2}=c \omega_{1}$.

Proof. If $n=1$, then the conclusion is obvious. Assume that $n>1$. Let $\mathcal{A}_{1}$ (resp. $\mathcal{A}_{2}$ ) be the set of all $\omega_{1}$ (resp. $\omega_{2}$ ) symplectic 2-dimensional subspaces of $X$. These sets are open and dense in the Grassmannian $G(2,2 n)$. Hence $\mathcal{A}_{1} \cap \mathcal{A}_{2} \neq \emptyset$. Take $H \in \mathcal{A}_{1} \cap \mathcal{A}_{2}$. We have $\mathcal{A}_{1}=\mathbf{S p}\left(X, \omega_{1}\right) H \subset$ $\operatorname{Sp}\left(X, \omega_{2}\right) H=\mathcal{A}_{2}$. Now apply Proposition 2.4 to $X=\left(X, \omega_{1}\right), Y=\left(X, \omega_{2}\right)$ and $F=$ identity.

3. Hamiltonian symplectomorphisms. Let $X=\left(\mathbb{R}^{2 n}, \omega_{0}\right)$ be the standard symplectic vector space. In $X$ we consider the norm $\left\|\left(a_{1}, \ldots, a_{2 n}\right)\right\|$ $=\max _{i=1}^{2 n}\left|a_{i}\right|$. Take a smooth function $H: X \times \mathbb{R} \ni(z, t) \mapsto H(z, t) \in \mathbb{R}$ and consider a system of differential equations (cf. [4])

$$
\frac{d}{d t} \phi(t, z)=J_{0}\left(\nabla_{z} H\right)(\phi(t, z), t), \quad \phi(0, z)=z .
$$

Assume that this system has a solution $\phi(t, z)$ for all $z$ and $t$ (this holds, e.g., if the supports of all functions $H_{t}, t \in \mathbb{R}$, are contained in a compact set). Then we can define the diffeomorphism

$$
\Phi(z)=\phi(1, z) .
$$

It is not difficult to check that $\Phi$ is a symplectomorphism.

Definition 3.1. Let $\Phi: X \rightarrow X$ be a symplectomorphism. We say that $\Phi$ is a hamiltonian symplectomorphism if it is given by the formula (3.1) for some smooth function $H$. We also say that $H$ is a Hamiltonian of $\Phi$.

Lemma 3.2. All elementary linear symplectomorphisms are hamiltonian symplectomorphisms.

Proof. Indeed:

- $L_{i}(c)$ is given by the Hamiltonian $H(x, y)=(c / 2) x_{i}^{2}$,

- $L_{i j}(c)$ is given by the Hamiltonian $H(x, y)=c x_{i} x_{j}$, 
- $R_{i}(c)$ is given by the Hamiltonian $H(x, y)=-(c / 2) y_{i}^{2}$,

- $R_{i j}(c)$ is given by the Hamiltonian $H(x, y)=-c y_{i} y_{j}$.

We notice that the hamiltonian flows generating the corresponding elementary symplectomorphisms are defined on the whole $\mathbb{R}^{2 n} \times \mathbb{R}$.

Now we show how to compute a Hamiltonian of a linear symplectomorphism:

TheOREM 3.3. Let $L: \mathbb{R}^{2 n} \rightarrow \mathbb{R}^{2 n}$ be a linear symplectomorphism. Then $L$ has a polynomial Hamiltonian

$$
H_{L}(z, t)=\sum_{i, j=1}^{2 n} a_{i, j}(t) z_{i} z_{j},
$$

where $a_{i, j}(t) \in \mathbb{R}[t]$ are polynomials of $t$. Moreover, we can compute $H_{L}$ effectively.

Proof. Let $L=L_{m} \circ \cdots \circ L_{1}$ where $L_{i}$ are elementary symplectomorphisms. We proceed by induction on $m$. If $m=1$ then we can use Lemma 3.2. In this case the flow $L_{1}(t)$ depends linearly on $t$.

Now consider $L^{\prime}=L_{m-1} \circ \cdots \circ L_{1}$. By the induction hypothesis $L^{\prime}(t)=$ $L_{m-1}(t) \circ \cdots \circ L_{1}(t)$ is given by a Hamiltonian $H^{\prime}$ of the form (3.2). Let $H^{\prime \prime}$ be the Hamiltonian of $L_{m}$ (as in Lemma 3.2). Now the flow $L(t)=L_{m}(t) \circ L^{\prime}(t)$ is given by the Hamiltonian

$$
H(z, t)=H^{\prime \prime}(z)+H^{\prime}\left(L_{m}(t)^{-1}(z), t\right) .
$$

Of course it has also the form (3.2). Since we can decompose $L$ into the product $L=L_{m} \circ \cdots \circ L_{1}$ effectively (see the proof of Theorem 2.1 ), we can also compute $H$ in an effective way.

Proposition 3.4. Let $L: \mathbb{R}^{2 n} \rightarrow \mathbb{R}^{2 n}$ be a hamiltonian symplectomorphism given by the flow $z \mapsto \phi(t, z), t \in \mathbb{R}$. Assume that $\phi(t, 0)=0$ for $t \in[0,1]$. For every $\eta>0$ there is an $\epsilon>0$ and a hamiltonian symplectomorphism $\Phi: \mathbb{R}^{2 n} \rightarrow \mathbb{R}^{2 n}$ such that

- $\Phi(z)=L(z)$ for all $z$ with $\|z\| \leq \epsilon$,

- $\Phi(z)=z$ for all $z$ with $\|z\| \geq \eta$.

Proof. We know that $L(z)=\phi(1, z)$, where $\phi(t, z)$ is the solution of some differential equation

$$
\frac{d}{d t} \phi(t, z)=J_{0}\left(\nabla_{z} H\right)(\phi(t, z), t), \quad \phi(0, z)=z .
$$

Since $\phi(t, 0)=0$ for every $t \in[0,1]$, we can find $\epsilon>0$ so small that all trajectories $\{\phi(t, z): 0 \leq t \leq 1\}$ which start from the ball $B(0, \epsilon)$ are contained in the ball $B(0, \eta / 2)$. Let $\sigma: \mathbb{R}^{2 n} \rightarrow \mathbb{R}$ be a smooth function such 
that

$$
\sigma(z)= \begin{cases}1 & \text { if }\|z\| \leq \eta / 2 \\ 0 & \text { if }\|z\| \geq \eta\end{cases}
$$

Take $S=\sigma H$. The hamiltonian symplectomorphism $\Phi$ given by the differential equation

$$
\frac{d}{d t} \phi(t, z)=J_{0}\left(\nabla_{z} S\right)(\phi(t, z), t), \quad \phi(0, z)=z,
$$

is well defined on the whole of $\mathbb{R}^{2 n}$ and

$$
\Phi(z)= \begin{cases}L(z) & \text { if }\|z\| \leq \epsilon, \\ z & \text { if }\|z\| \geq \eta .\end{cases}
$$

Now Theorem 3.3 easily yields the following:

COROLlary 3.5. Let $L: \mathbb{R}^{2 n} \rightarrow \mathbb{R}^{2 n}$ be a linear symplectomorphism. For every $\eta>0$ there is an $\epsilon>0$ and a hamiltonian symplectomorphism $\Phi: \mathbb{R}^{2 n} \rightarrow \mathbb{R}^{2 n}$ such that

- $\Phi(z)=L(z)$ for all $z$ with $\|z\| \leq \epsilon$,

- $\Phi(z)=z$ for all $z$ with $\|z\| \geq \eta$.

4. Characterization of symplectomorphisms. Before we formulate our next result we need the following (well-known)

LEMMA 4.1. Let $X=\left(\mathbb{R}^{2 n}, \omega_{0}\right)$ be the standard symplectic vector space. Fix $\eta>0$ and let $a, b \in B(0, \eta)$. Then there exists a symplectomorphism $\Phi: X \rightarrow X$ such that

$$
\Phi(a)=b \quad \text { and } \quad \Phi(z)=z \quad \text { for }\|z\| \geq 2 \eta .
$$

Proof. Let $c=\left(c_{1}, \ldots, c_{2 n}\right)=b-a$. Define a sequence of points as follows:

$$
a_{0}=a, \quad a_{i}=a_{i-1}+\left(0, \ldots, 0, c_{i}, 0, \ldots, 0\right) .
$$

Of course $a_{i} \in B(0, \eta)$ and $a_{2 n}=b$. Now consider the translation

$$
T_{i}: \mathbb{R}^{2 n} \ni(x, y) \mapsto(x, y)+\left(0, \ldots, 0, c_{i}, 0, \ldots, 0\right) \in \mathbb{R}^{2 n} .
$$

We have $T_{i}\left(a_{i-1}\right)=a_{i}$ for $i=1, \ldots, 2 n$.

The translation $T_{i}$ is a hamiltonian symplectomorphism given by the Hamiltonian

$$
H_{i}(x, y)= \begin{cases}-c_{i} y_{i} & \text { if } i \leq n \\ c_{i} x_{i-n} & \text { if } i>n .\end{cases}
$$

Let $V_{i}$ be the symplectic vector field which is determined by the Hamiltonian $H_{i}$. Since the ball $B(0, r)$ is a convex set, all trajectories $\phi(t), 0 \leq t \leq 1$, of the symplectic vector fields $V_{i}$ which begin at $a_{i}$ lie in the ball $B(0, \eta)$. 
Let $\sigma: \mathbb{R}^{2 n} \rightarrow \mathbb{R}$ be a smooth function such that

$$
\sigma(z)= \begin{cases}1 & \text { if }\|z\| \leq \eta \\ 0 & \text { if }\|z\| \geq 2 \eta\end{cases}
$$

Now let $F_{i}: \mathbb{R}^{2 n} \rightarrow \mathbb{R}^{2 n}$ be the hamiltonian symplectomorphism given by the Hamiltonian $G_{i}=\sigma H_{i}$. Then

$$
G_{i}\left(a_{i-1}\right)=a_{i} \quad \text { and } \quad G_{i}(z)=z \quad \text { if }\|z\| \geq 2 \eta .
$$

Now it is enough to take $\Phi=G_{2 n} \circ G_{2 n-1} \circ \cdots \circ G_{1}$.

We apply Proposition 3.4 to the general case:

TheOrem 4.2. Let $(X, \omega)$ be a symplectic manifold. Let $a_{1}, \ldots, a_{m}$ and $b_{1}, \ldots, b_{m}$ be two families of points of $X$. For every $i=1, \ldots, n$ choose a linear symplectomorphism $L_{i}: T_{a_{i}} X \rightarrow T_{b_{i}} X$. Then there is a symplectomorphism $\Phi: X \rightarrow X$ such that

$$
\Phi\left(a_{i}\right)=b_{i}, \quad d_{a_{i}} \Phi=L_{i}, \quad i=1, \ldots, m .
$$

Proof. By the Darboux Theorem every point $z \in X$ has an open neighborhood $V_{z}$ which is symplectically isomorphic to the ball $B\left(0, r_{z}\right)$ in the standard vector space $\left(\mathbb{R}^{2 n}, \omega_{0}\right)$. Denote by $U_{z} \subset V_{z}$ the open set which corresponds to the ball $B\left(0, r_{z} / 3\right)$.

Since $\operatorname{dim} X \geq 2$ the manifold $X \backslash\left\{a_{2}, \ldots, a_{m}\right\}$ is also connected. Hence there exists a smooth path $\gamma: I \rightarrow X$ such that $\gamma(0)=a_{1}, \gamma(1)=b_{1}$ and $\left\{a_{2}, \ldots, a_{m}\right\} \cap \gamma(I)=\emptyset$. Additionally we can assume that the sets $V_{z}$ which cover $\gamma(I)$ are also disjoint from $\left\{a_{2}, \ldots, a_{m}\right\}$.

Let $\epsilon$ be a Lebesgue number for the function $\gamma: I \rightarrow X$ with respect to the cover $\left\{U_{z}\right\}_{z \in X}$ and choose an integer $N$ with $1 / N<\epsilon$. If $I_{k}:=$ $[k / N,(k+1) / N]$, then $\gamma\left(I_{k}\right)$ is contained in some $U_{z}$; denote it by $U_{k}$, the set $V_{z}$ by $V_{k}$, and $r_{z}$ by $r_{k}$. Let $A_{k}:=\gamma(k / N)$, in particular $A_{0}=a_{1}, A_{N}=b_{1}$.

Since $V_{k} \cong B\left(0, r_{k}\right)$ and $A_{k}, A_{k+1} \in B\left(0, r_{k} / 3\right)$ we can apply Lemma 4.1 to obtain a symplectomorphism $\Phi: B\left(0, r_{k}\right) \rightarrow B\left(0, r_{k}\right)$ such that

$$
\Phi\left(A_{k}\right)=A_{k+1} \quad \text { and } \quad \Phi(z)=z \quad \text { for }\|z\| \geq(2 / 3) r_{k} .
$$

We can extend $\Phi$ to the whole of $X$ (we glue it with the identity); denote this extension by $\Phi_{k}$. Put

$$
\Psi=\Phi_{N} \circ \Phi_{N-1} \circ \cdots \circ \Phi_{0} .
$$

Then $\Psi\left(a_{1}\right)=b_{1}$ and $\Psi\left(a_{i}\right)=a_{i}$ for $i>1$. Repeating this process, we finally arrive at a symplectomorphism $\Sigma: X \rightarrow X$ such that $\Sigma\left(a_{i}\right)=b_{i}$ for $i=1, \ldots, m$. In a similar way using Proposition 3.5 we can construct a symplectomorphism $\Pi: X \rightarrow X$ such that

$$
\Pi\left(b_{i}\right)=b_{i}, \quad d_{b_{i}} \Pi=L_{i} \circ\left(d_{a_{i}} \Sigma\right)^{-1} .
$$

Now it is enough to take $\Phi=\Pi \circ \Sigma$. 
Since for a compact symplectic manifold $(X, \omega)$ of dimension $2 n$ it is well known that for a fixed number $0<s \leq n$ there exists a closed $2 s$-dimensional symplectic submanifold $Z \subset X$ (which is a nontrivial result by S. Donaldson [3, Corollary 6, p. 671]), we can use Theorem 4.2 to obtain:

Corollary 4.3. Let $(X, \omega)$ be a compact symplectic manifold of dimension $2 n$. Let $a_{1}, \ldots, a_{m}$ be a family of points of $X$. Take $0<s \leq n$. For every $i=1, \ldots, m$ choose a linear $2 s$-dimensional symplectic subspace $H_{i} \subset T_{a_{i}} X$. Then there is a closed symplectic $2 s$-dimensional submanifold $Y \subset X$ such that

$$
a_{i} \in Y, \quad T_{a_{i}} Y=H_{i}, \quad i=1, \ldots, m .
$$

In contrast to the previously mentioned result on existence of symplectic submanifolds, the existence of isotropic tori is easy to prove in Darboux local coordinates. In a similar way to Corollary 4.3 we get:

Corollary 4.4. Let $(X, \omega)$ be a symplectic manifold of dimension $2 n$. Let $a_{1}, \ldots, a_{m}$ be a family of points of $X$. Take $0<k \leq n$. For every $i=1, \ldots, m$ choose a linear $k$-dimensional isotropic subspace $H_{i} \subset T_{a_{i}} X$. Then there is a closed isotropic $k$-dimensional torus $Y \subset X$ such that

$$
a_{i} \in Y, \quad T_{a_{i}} Y=H_{i}, \quad i=1, \ldots, m .
$$

5. Diffeomorphisms that are symplectomorphisms. Finally, we show that a symplectomorphism can be described as a diffeomorphism which preserves symplectic or isotropic submanifolds of a given fixed dimension.

TheOREM 5.1. Let $\left(X, \omega_{X}\right)$ and $\left(Y, \omega_{Y}\right)$ be compact symplectic manifolds of dimension $2 n>2$. Fix a number $0<s<n$. Assume that $\Phi: X \rightarrow Y$ is a diffeomorphism which maps all $2 s$-dimensional symplectic submanifolds of $X$ onto symplectic submanifolds of $Y$. Then $\Phi$ is a conformal symplectomorphism, i.e., there exists a non-zero number $c \in \mathbb{R}$ such that

$$
\Phi^{*} \omega_{Y}=c \omega_{X} .
$$

Proof. Fix $z \in X$ and let $H \subset T_{z} X$ be a $2 s$-dimensional symplectic subspace of $T_{z} X$. By Corollary 4.3 (applied for $m=1, a_{1}=z$ and $H_{1}=H$ ) there exists a $2 s$-dimensional symplectic submanifold $M$ of $X$ such that $z \in M$ and $T_{z} M=H$.

Let $\Phi(M)=M^{\prime}$ and $z^{\prime}=\Phi(z)$. By assumption the submanifold $M^{\prime} \subset Y$ is symplectic. This means that the space $d_{z} \Phi(H)=T_{z^{\prime}} M^{\prime}$ is symplectic. Hence $d_{z} \Phi$ maps all linear $2 s$-dimensional symplectic subspaces of $T_{z} X$ onto subspaces of the same type. By Proposition 2.4 this implies that $d_{z} \Phi$ is a conformal symplectomorphism. i.e.,

$$
\left(d_{z} \Phi\right)^{*} \omega_{Y}=\lambda(z) \omega_{X},
$$


where $\lambda(z) \neq 0$. This means that there is a smooth function $\lambda: X \rightarrow \mathbb{R}^{*}$ $(=\mathbb{R} \backslash\{0\})$ such that

$$
\Phi^{*} \omega_{Y}=\lambda \omega_{X} .
$$

But since the form $\omega_{Y}$ is closed, so is $\Phi^{*} \omega_{Y}$. Since $n>1$ this implies that the derivative $d \lambda$ vanishes, i.e., the function $\lambda$ is constant.

TheOREM 5.2. Let $\left(X, \omega_{X}\right)$ and $\left(Y, \omega_{Y}\right)$ be symplectic manifolds of dimension $2 n>2$. Fix a number $1<k \leq n$. Assume that $\Phi: X \rightarrow Y$ is a diffeomorphism which maps all $k$-dimensional isotropic tori of $X$ onto isotropic tori of $Y$. Then $\Phi$ is a conformal symplectomorphism, i.e., there exists a non-zero constant $c \in \mathbb{R}$ such that

$$
\Phi^{*} \omega_{Y}=c \omega_{X} .
$$

Proof. Fix $z \in X$ and let $H \subset T_{z} X$ be a $k$-dimensional isotropic subspace of $T_{z} X$. By Corollary 4.4 (applied for $m=1, a_{1}=z$ and $H_{1}=H$ ) there exists a $k$-dimensional isotropic torus $M$ of $X$ such that $z \in M$ and $T_{z} M=H$.

Let $\Phi(M)=M^{\prime}$ and $z^{\prime}=\Phi(z)$. By assumption the torus $M^{\prime} \subset Y$ is isotropic. This means that the space $d_{z} \Phi(H)=T_{z^{\prime}} M^{\prime}$ is isotropic. Hence $d_{z} \Phi$ maps all linear $k$-dimensional isotropic subspaces of $T_{z} X$ onto subspaces of the same type. By Proposition 2.5 this implies that $d_{z} \Phi$ is a conformal symplectomorphism. The rest of the proof is the same as in the case of Theorem 5.1 above.

REMARK 5.3. Let us note that in particular if $\Phi$ maps Lagrangian tori onto tori of the same type then $\Phi$ is a conformal symplectomorphism.

Corollary 5.4. Let $X$ be a compact manifold of dimension $2 n>2$. Let $\omega_{1}$ and $\omega_{2}$ be two symplectic forms on $X$. Fix a number $0<k<n$. Assume that every $2 k$-dimensional $\omega_{1}$-symplectic submanifold of $X$ is $\omega_{2}$-symplectic. Then there exists a non-zero number $c \in \mathbb{R}$ such that

$$
\omega_{1}=c \omega_{2}
$$

Proof. It is enough to apply Theorem 5.1 to $X=\left(X, \omega_{1}\right), Y=\left(X, \omega_{2}\right)$ and $\Phi=$ identity.

Corollary 5.5. Let $(X, \omega)$ be a compact symplectic manifold of dimension $2 n>2$. Fix a number $0<k<n$. Assume that $\Phi: X \rightarrow X$ is a diffeomorphism which maps all $2 k$-dimensional symplectic submanifolds of $X$ onto submanifolds of the same type. Then $\Phi$ is a symplectomorphism or an antisymplectomorphism, i.e., $\Phi^{*} \omega= \pm \omega$. If $\Phi$ preserves orientation and $n$ is odd, then $\Phi$ is a symplectomorphism. Moreover, if $n$ is even, then $\Phi$ has to preserve orientation. 
Proof. Indeed, we have $\Phi^{*} \omega=c \omega$. We can write

$$
\operatorname{vol}(X)=\int_{X} \omega^{n}= \pm \int_{X} \Phi^{*} \omega^{n}= \pm c^{n} \int_{X} \omega^{n},
$$

hence $c= \pm 1$. Moreover, if $\Phi$ preserves orientation and $n$ is odd, then we get $c=1$. If $n$ is even then $(-\omega)^{n}=\omega^{n}$ and $\Phi$ has to preserve orientation.

Remark 5.6. Corollaries like Corollary 5.4 and Corollary 5.5 are also true for a compact symplectic manifold $X$ in the case of isotropic tori. A similar geometric characterization of symplectomorphisms has already been proved for diffeomorphisms preserving capacity, which turn out to be symplectic or antisymplectic (cf. [5],[8]).

EXAMPLE 5.7. We show that in the general case $\Phi$ need not to be a symplectomorphism. Let $Y=\left(S^{2}, \omega\right)$ (where $\omega$ is the standard volume form on the sphere) and let $\left(X_{n}, \omega_{n}\right)=\prod_{i=1}^{n} Y$ be a standard symplectic product. Further, let $\sigma: S^{2} \ni(x, y, z) \mapsto(x, y,-z) \in S^{2}$ be a mirror symmetry. Of course $\sigma^{*} \omega=-\omega$. More generally, if $\Sigma=\prod_{i=1}^{n} \sigma: X_{n} \rightarrow X_{n}$, then $\Sigma^{*} \omega_{n}$ $=-\omega_{n}$. Hence $\Phi$ from Corollary 5.5 may well be an antisymplectomorphism.

However, in any case either $\Phi$ or $\Phi \circ \Phi$ is a symplectomorphism.

Now let $(X, \omega)$ be a symplectic manifold and denote by $\operatorname{Symp}(X, \omega)$ the group of symplectomorphisms of $X$. To end this note we show that this group also determines the conformal symplectic structure of $X$ :

TheOREM 5.8. Let $X$ be a smooth manifold of dimension $2 n>2$ and let $\omega_{1}, \omega_{2}$ be two symplectic forms on $X$. If $\operatorname{Symp}\left(X, \omega_{1}\right) \subset \operatorname{Symp}\left(X, \omega_{2}\right)$, then there exists a non-zero constant $c$ such that $\omega_{2}=c \omega_{1}$.

Proof. Take $z \in X$ and consider the symplectic vector spaces $V_{1}=$ $\left(T_{z} X, \omega_{1}\right)$ and $V_{2}=\left(T_{z} X, \omega_{2}\right)$. By Theorem 4.2 , for every linear symplectomorphism $S$ of $V_{1}$, there is a symplectomorphism $\Phi_{S} \in \operatorname{Symp}\left(X, \omega_{1}\right)$, such that

$$
\Phi_{S}(z)=z, \quad d_{z} \Phi_{S}=S .
$$

Since $\operatorname{Symp}\left(X, \omega_{1}\right) \subset \operatorname{Symp}\left(X, \omega_{2}\right)$ we easily obtain $\mathbf{S p}\left(V_{1}\right) \subset \mathbf{S p}\left(V_{2}\right)$. Consequently, by Proposition 2.6 there exists a non-zero number $\lambda(z)$ such that $\omega_{2}(z)=\lambda(z) \omega_{1}(z)$. Now we finish the proof as in the proof of Theorem 5.1.

Acknowledgements. This research was partially supported by EC FP6 Marie Curie ToK programme SPADE2, MTKD-CT-2004-014508 and Polish MNiSW SPB-M. 


\section{References}

[1] A. Borel, Injective endomorphisms of algebraic Nash varieties, Arch. Math. (Basel) 20 (1969), 531-537.

[2] E. Calabi, On the group of automorphisms of a symplectic manifold, in: Problems in Analysis, R. Gunning (ed.), Princeton Univ. Press, 1970, 1-26.

[3] S. Donaldson, Symplectic submanifolds and almost-complex geometry, J. Differential Geom. 44 (1996), 666-705.

[4] I. Ekeland and H. Hofer, Symplectic topology and Hamiltonian dynamics, Math. Z. 200 (1989), 355-378.

[5] H. Hofer, Dynamics, topology and holomorphic curves, Documenta Math., Extra volume ICM, Vol. II (1998), 443-452.

[6] S. Janeczko and Z. Jelonek, Linear automorphisms that are symplectomorphisms, J. London Math. Soc. 69 (2004), 503-517.

[7] D. McDuff, A survey of topological properties of groups of symplectomorphisms, in: Topology, Geometry and Quantum Field Theory, Proc. of 2002 Symposium in honor of G. B. Segal, ed. U. L. Tillmann, Cambridge Univ. Press, 2004, 173-193.

[8] D. McDuff and D. Salamon, Introduction to Symplectic Topology, 2nd ed., Oxford Univ. Press, 1998.

[9] L. Polterovich, The Geometry of the Group of Symplectic Transformations, Lecture Notes in Math. ETH Zürich, Birkhäuser, 2001.

[10] I. Vaisman, Locally conformal symplectic manifolds, Int. J. Math. Sci. 8 (1985), $521-536$.

[11] A. Weinstein, Lectures on Symplectic Manifolds, CBMS Reg. Conf. Ser. Math. 29, Amer. Math. Soc., Providence RI, 1977.

S. Janeczko

Instytut Matematyczny PAN

Śniadeckich 8

00-956 Warszawa, Poland

and

Wydział Matematyki i Nauk Informacyjnych

Politechnika Warszawska

Pl. Politechniki 1

00-661 Warszawa, Poland

E-mail: janeczko@mini.pw.edu.pl
Z. Jelonek Instytut Matematyczny PAN

Śniadeckich 8 00-956 Warszawa, Poland E-mail: najelone@cyf-kr.edu.pl

Received 17 September 2008;

in revised form 2 March 2009 